\title{
The Physiology of Pregnancy lllustrated with Ultrasound Images
}

\author{
${ }^{1}$ Herbert F Janssen, ${ }^{2}$ David L Osborne, ${ }^{3}$ Sanja Plavsic Kupesic
}

${ }^{1}$ Professor of Physiology, Department of Medical Education and Department of Orthopedic Surgery and Rehabilitation, Paul L Foster School of Medicine, Texas Tech University Health Sciences Center, Texas Tech University Health Sciences Center 5001 El Paso Drive, El Paso, Texas 79905, USA

${ }^{2}$ Professor of Physiology, Department of Medical Education, Paul L Foster School of Medicine, Texas Tech University Health Sciences Center, 5001 El Paso Drive, El Paso, Texas 79905, USA

${ }^{3}$ Professor and Clinical Professor of Obstetrics and Gynecology and Radiology, Department of Medical Education, Paul L Foster School of Medicine, Texas Tech University Health Sciences Center, 5001 El Paso Drive, El Paso, Texas 79905, USA

Correspondence: Herbert F Janssen, Professor of Physiology, Department of Medical Education and Department of Orthopedic Surgery and Rehabilitation, Paul L Foster School of Medicine, Texas Tech University Health Sciences Center, Texas Tech University Health Sciences Center, 5001 El Paso Drive, El Paso, Texas 79905, USA, e-mail: herb.janssen@ttuhsc.edu

\begin{abstract}
Pregnancy is the culmination of many unexplained anatomical, physiological, and biochemical processes. While many aspects of pregnancy remain vague, ultrasound imaging techniques have provided a clearer understanding of many aspects of the process. This has included a more complete description of the ovulation process and implantation of the blastocyst into the endometrium. Ultrasound imaging using 3-D and 4-D capabilities has also provided a dynamic view of fetal development. A clearer and more complete description of fetal circulation is now available. In the hands of trained individuals, ultrasound techniques have captured fetal heart images revealing both normal and abnormal development. As the techniques improve, this progression of knowledge will also increase our ability to understand the entire reproductive process.
\end{abstract}

Keywords: Ultrasound, pregnancy, fetal development, blood flow, fetal circulation.

\section{INTRODUCTION}

Philosophers have marveled at the power the female reproductive system exerts over human existence. Physiologists have unsuccessfully attempted to unravel the web of neural and hormonal control mechanisms that influence this process. Physicians continue to correlate the art and science of medicine as they help patients deal with the emotional, psychological, and physical complexities associated with reproduction. It is unlikely any of these disciplines will be able to complete their quest any time in the foreseeable future.

A successful pregnancy is the ultimate role played by the female's reproductive organs. Anatomical images produced by modern ultrasound have provided an opportunity to correlate structure with physiological changes in reproductive hormones and other control mechanisms. A pregnancy resulting in a successful delivery represents fulfillment of nature's goal to continue the species. The complexity of the process continues to amaze everyone from layperson to scientist and from philosopher to physician. Starting with conception and ending with a newly born infant, the entire process is nothing short of a miracle. Ultrasound imaging devices have provided scientists and physicians with the first truly useful tool capable of providing images of the developing embryo. Its noninvasive qualities have repeatedly been shown safe, even for the fragile, developing tissues. Dating back to the seemingly primitive B-mode units, the ultrasound images have provided important medical information for the physician and smiles and reassurance to the mother. The explosion in technology has recently provided 3-D and 4-D ultrasound images that produce real-time images of newly developing life. These units, equipped with improved computer hardware, advanced software, and newly designed ultrasound components, continue to astonish. The advancement in capability seems endless, and the improvements are being introduced at a rate that would have been unachievable even a few years ago. 


\section{ULTRASOUND IMAGES OF THE REPRODUCTIVE ORGANS}

Ultrasound images of the female reproductive organs have provided a clearer understanding of the normal structures and greatly enhanced our ability to diagnose pathological conditions. These images have also allowed us to better understand the physiology of the reproductive process. The illustrations that follow demonstrate these capabilities. The 3-D images of the ovaries show the development of the follicle at the time of its release. While beyond the scope of this article, the ultrasound images can also reveal pathological changes in the ovary. When combined with laboratory values of reproductive hormones, a skilled physician can use ultrasound to evaluate conditions that may prevent pregnancy.

\section{PHYSIOLOGICAL AND ANATOMICAL CONSIDERATIONS LEADING UP TO BLASTOCYST IMPLANTATION}

Once released, the ovum moves to the awaiting opening of the ampulla where it is collected and starts its travel down the fallopian tube (oviduct). If present, sperm delivered by the male combines with the ovum in the distal region of this tube. The interaction of the sperm and ovum prior to a successful fertilization is a relatively complex process that requires preparation of both for the union. The head of the sperm must be altered so that it can penetrate the ovum as their paths cross. Likewise, the ovum must not allow more than one sperm to cross its outer barrier. After one and only one sperm fertilizes the ovum, cell division starts quickly. The fertilized ovum reaches upwards of 100 cells before the blastocyst reaches the uterus some 5-7 days later. Movement of the dividing mass of cells down the fallopian tube is facilitated by fluid in the tube and the motion of ciliated epithelium. Contractions of the tube may also play a role in this movement.

The vascular rich lining of the uterus, the endometrium, has been awaiting arrival and implantation of the blastocyst. If implantation of the blastocyst does not occur, the endometrium is sloughed as menstrual fluid; however, if the blastocyst successfully implants, the uterus becomes the home for the developing embryo. During the next 38 weeks, the cluster of 100 cells develops into a full term fetus. Continuation of this structure is dependent on elevated levels of progesterone in the circulation plasma.

\section{THE INITIAL STAGES OF EMBRYO DEVELOPMENT}

The blastocyst contains an inner mass of cells that will later become the fetus. The outer layer or trophoblast forms the attachment to the vascular rich lining of the uterus. Cells of the trophoblast develop into the syncytial trophoblast which destroys the adjacent decidual cells allowing the blastocyst to penetrate deeper into the endometrium. ${ }^{1}$ This process continues until the blastocyst is completely embedded. The products released from the destroyed decidual cells help to increase hyperemia and capillary permeability. This provides a nutrition source for the developing cells until a placenta can be formed. The syncytial trophoblast and cytotrophoblast layer surrounding the blastocyst sends out columns of cells that serve to anchor the embryo. Later these columns differentiate into the placental villi. By the third week, the villi are invaded by the fetal blood vessels. This is the start of a primitive yet functional circulatory system (Figs 1A and B).

Development of this placental circulation is obviously essential for the continued development of the embryo. By the 16th day after fertilization the heart of the developing embryo is capable of pumping blood. This familiar sound can be heard in the physician's office with the assistance of inexpensive, hand-held Doppler ultrasound devices. More advanced ultrasound equipment can provide images of the heart and entire circulatory system, assuring the mother and physician of the embryo's continued development (Figs 2 and 3).

Simultaneously, blood sinuses supplied from the mother's circulation develop around the growing embryo. Nutrients supplied in this manner supplement those obtained from the digested deciduous. ${ }^{2}$ During this transitional phase the placenta must develop rapidly to insure the needed supply of nutrients and oxygen. Since blood is not exchanged between the mother and the developing embryo, the exchange of oxygen is totally dependent on diffusion across the placental membrane. This requires an adequate surface area and blood supply from both the mother and the fetus. Ultrasound can be used to monitor this process.

The process described above is dependent on the continued maintenance of the endometrium that would have been sloughed at the end of the menstrual cycle if implantation had not occurred. If the wall of the uterus were to slough after implantation occurred, the pregnancy would obviously be terminated. Human chorionic 


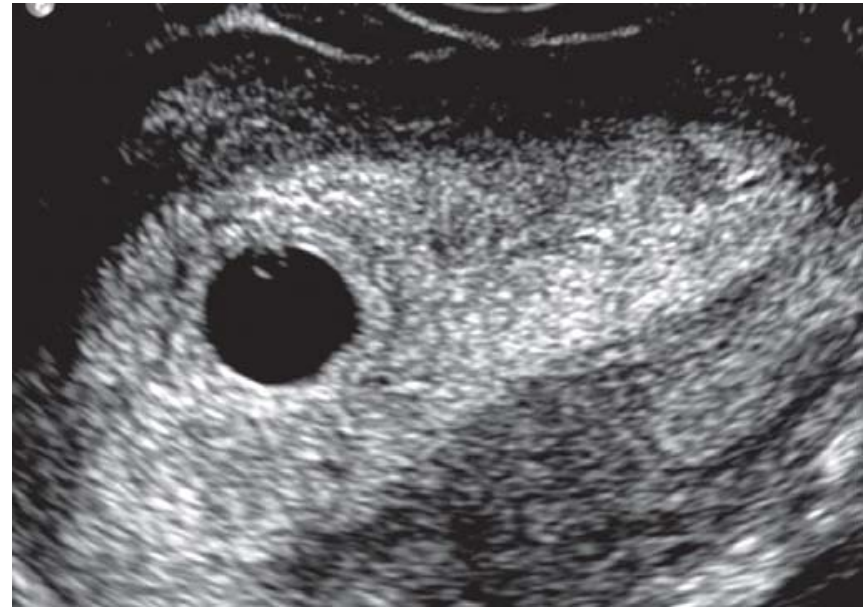

Fig. 1A: Transvaginal ultrasound of an early intrauterine gestational sac

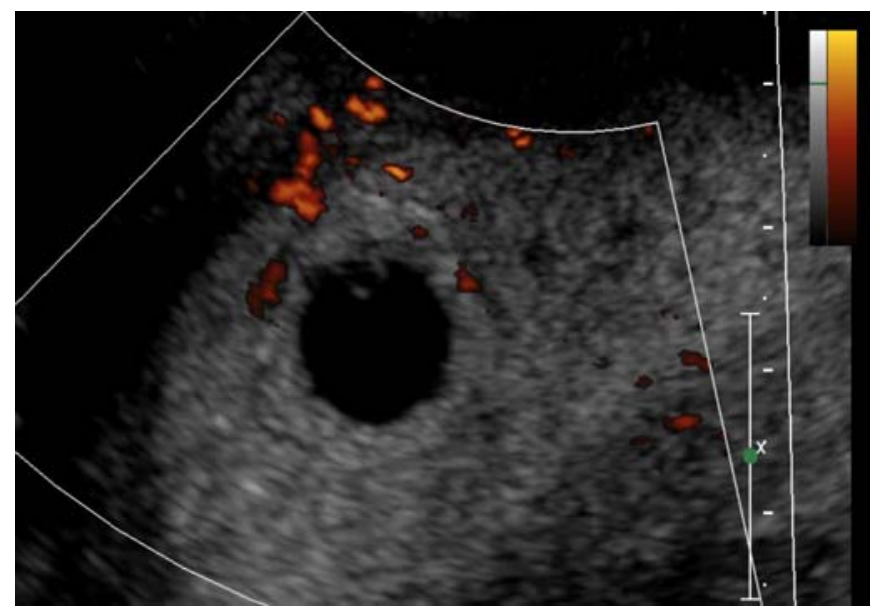

Fig. 1B: The same patient. Transvaginal power Doppler scan of a gestational sac at 5 weeks' gestation. This technique easily depicts blood flow surrounding the gestational sac

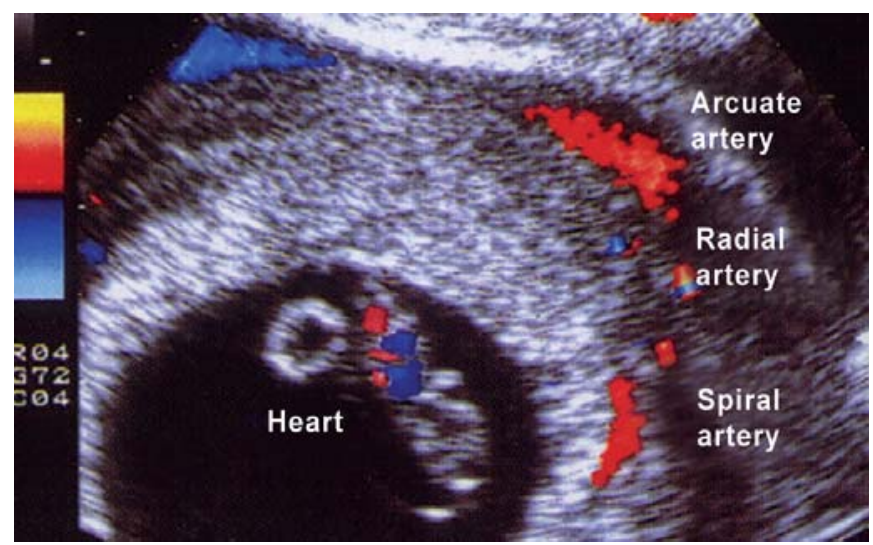

Fig. 2: Transvaginal color Doppler scan of a pregnancy at $7 / 8$ weeks' gestation. Sensitive color Doppler devices allow simultaneous visualization of the fetal, intervillous and uterine circulations

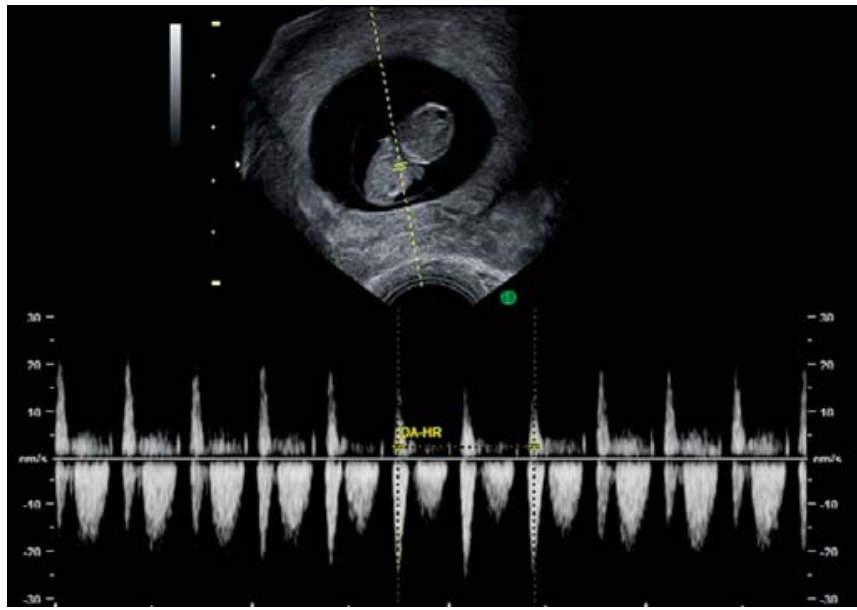

Fig. 3: Pulsed Doppler waveform analysis of the fetal heart action at 8 weeks' gestation

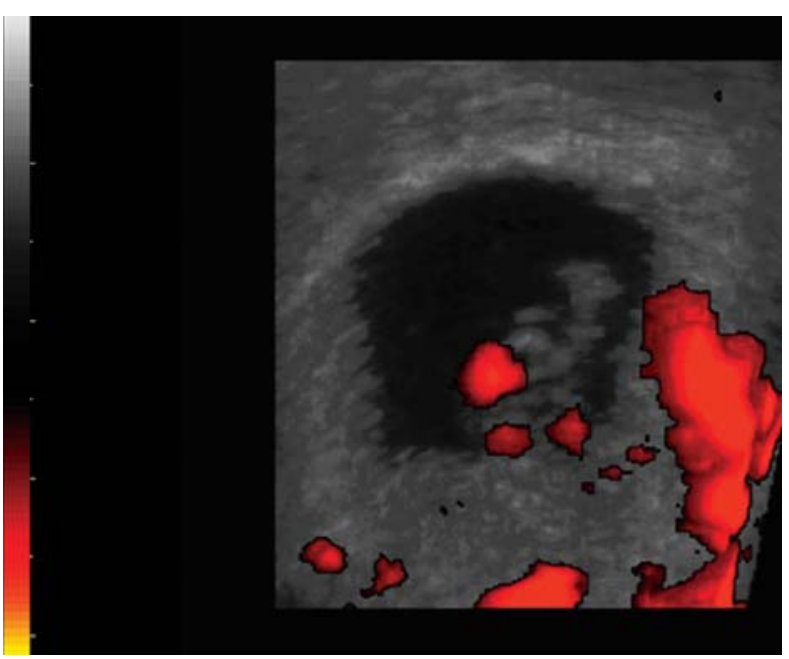

Fig. 4: Three-dimensional power Doppler image of embryonic and intervillous circulations at 7 weeks' gestation

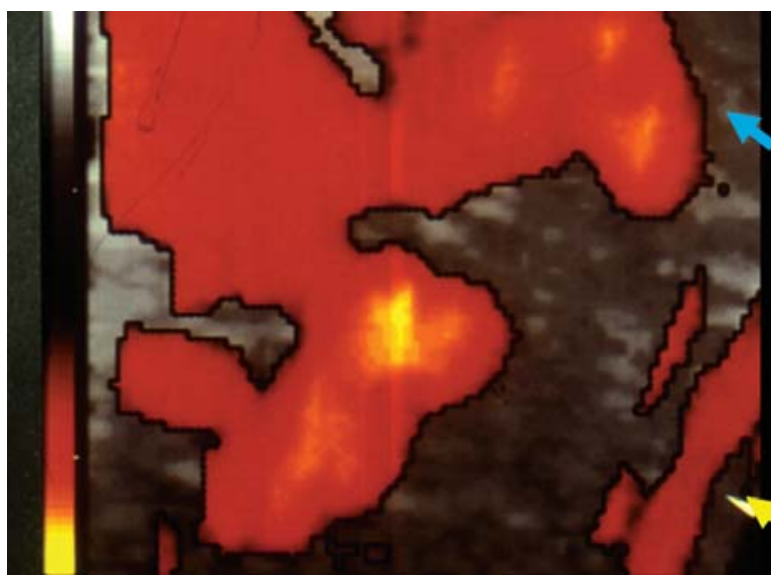

Fig. 5: Demonstration of the spiral arteries (blue arrow) and intervillous circulation (yellow arrow) by three-dimensional power Doppler 
gonadotropin secreted by the syncytial trophoblast into the fluids of the mother prevents this from occurring. This hormone, which can be detected in the blood within several days after implantation, prevents involution of the corpus luteum. Under the influence of human chorionic gonadotropin, the corpus luteum grows to approximately twice its size and continues to secrete estrogen and progesterone. Without these hormones from the corpus luteum, spontaneous abortion will always occur up to the 7 th week of pregnancy and possibly up to the 12th week. After that, the placenta will be secreting adequate amounts of the hormones to maintain pregnancy.

Human chorionic gonadotropin also plays an important role in the development of the male fetus. This hormone stimulates the production of testosterone in the male fetus and is essential to the development of male sex organs instead of female. Although human chorionic gonadotropin levels in the blood decline after reaching peak levels within 10-12 weeks, they remain elevated throughout pregnancy.

\section{DEVELOPMENT AND FUNCTION OF FETAL CIRCULATION DURING PREGNANCY}

Embryo development is not the focus of this article but maturation of the heart, the fetal circulation, and fetal blood directly alters the course of the pregnancy dramatically. The function of most other organs is totally, or at least partially, supplemented by the mother's system; however, a functioning heart must develop as the fetus grows. The fetus gains nutrients and oxygen from the mother through the placenta and loses its waste byproducts to the mother through this same route. Accomplishment of this task is dependent on an appropriate fetal and placental circulation (Figs 4 and 5). Like any multicellular organism with a closed vascular system, the developing fetus must have a mechanism to move blood for this to occur. The heart, RBC's, and vascular tree must keep pace with the developing fetal structures.

\section{THE HEART}

The heart starts to form in the first several weeks after fertilization. At the fourth week, the heart will begin to beat regularly. As early as the 3rd week, blood from the heart is pumped into vessels. ${ }^{3}$ The heart starts to develop the septal walls during the 4th and 5th weeks. Heart valves form between the 5th and 8th weeks. During the 3rd month, the

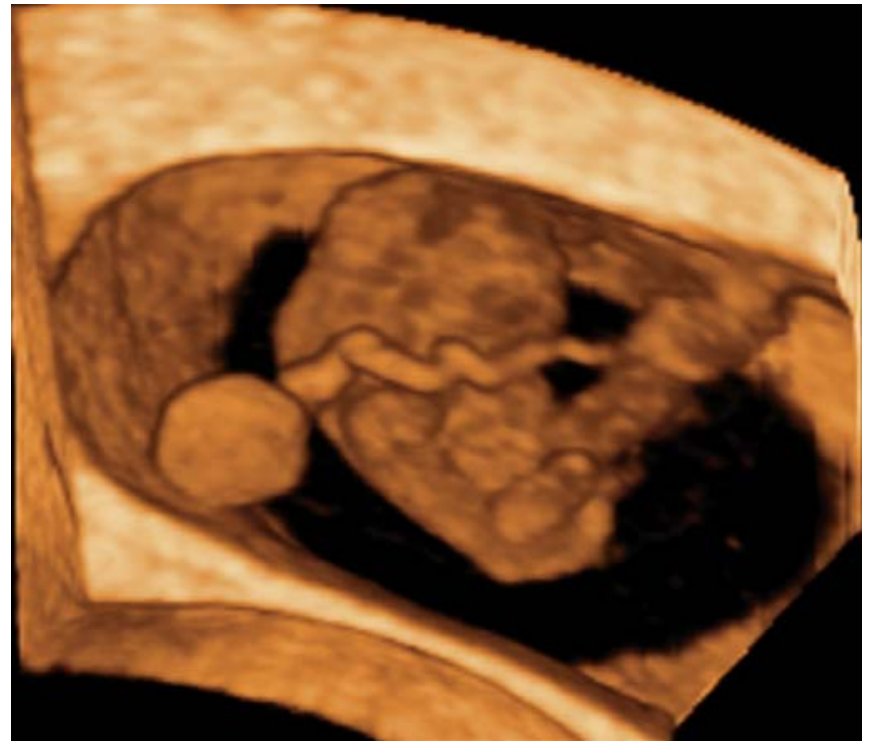

Fig. 6: Three-dimensional ultrasound of an embryo and yolk sac at 7/8 weeks' gestation. (From Embryology Image Library at Paul L Foster School of Medicine, Editors: Sanja Plavsic Kupesic, MD, PhD and Guillermo Azumendi, MD)

valve leaflets function to direct blood flow through the heart in the proper direction, preventing back-flow during ventricular contraction. ${ }^{3}$

\section{RED BLOOD CELLS (RBC's)}

Nucleated red blood cells (RBC's) are formed in the yolk sac and mesothelial layers of the placenta during the first weeks of embryo development (Fig. 6). At six weeks, the liver starts to make RBC's, and at 3 months the spleen and other lymphoid tissues take over this function. Gradually the bone marrow will become the supplier of RBC's for the fetal circulation. ${ }^{1}$

\section{CIRCULATION}

Blood from the heart is pumped into the primitive aortic arch and is returned through a venous system that will remodel later. By the 4th week, an early vascular system has developed. Oxygenated blood from the placenta is delivered to the heart by a pair of umbilical veins. This blood is initially pumped from the heart into the right and left aortic arches. Later these fuse to form a single midline dorsal aorta. Venous blood from the embryo is returned to the heart by six vessels. ${ }^{3}$ These consist of vessels that drain the anterior and posterior portions of the fetus (Figs 7 to 9).

Continued development of the blood vessels is promoted by the need for additional nutrients and oxygen by the growing tissue. Vascular endothelial growth factor (VEGF) 


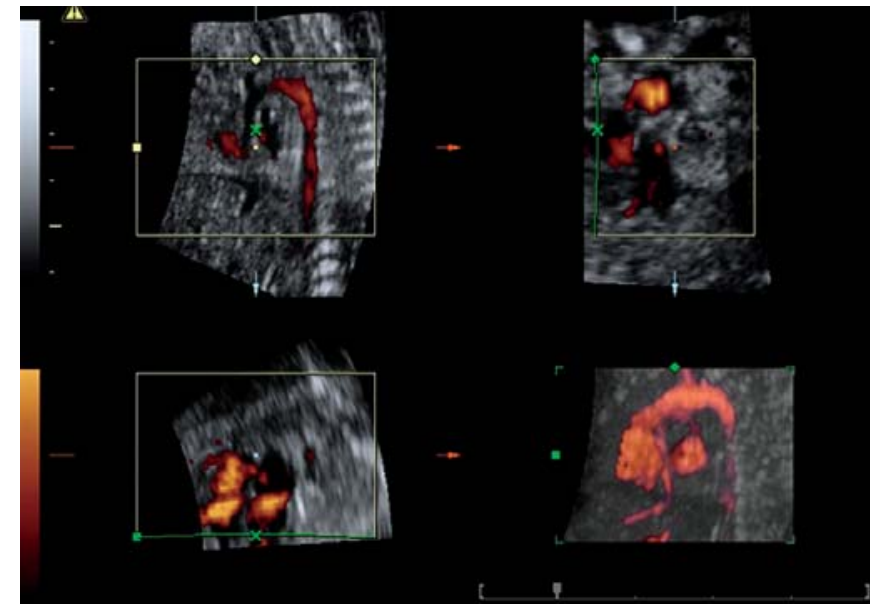

Fig. 7: Three-dimensional power Doppler scan of a fetus at 12 weeks' gestation. Fetal heart and arch of the aorta are clearly displayed. (From Embryology Image Library at Paul L Foster School of Medicine, Editors: Sanja Plavsic Kupesic, MD, PhD and Guillermo Azumendi, MD)

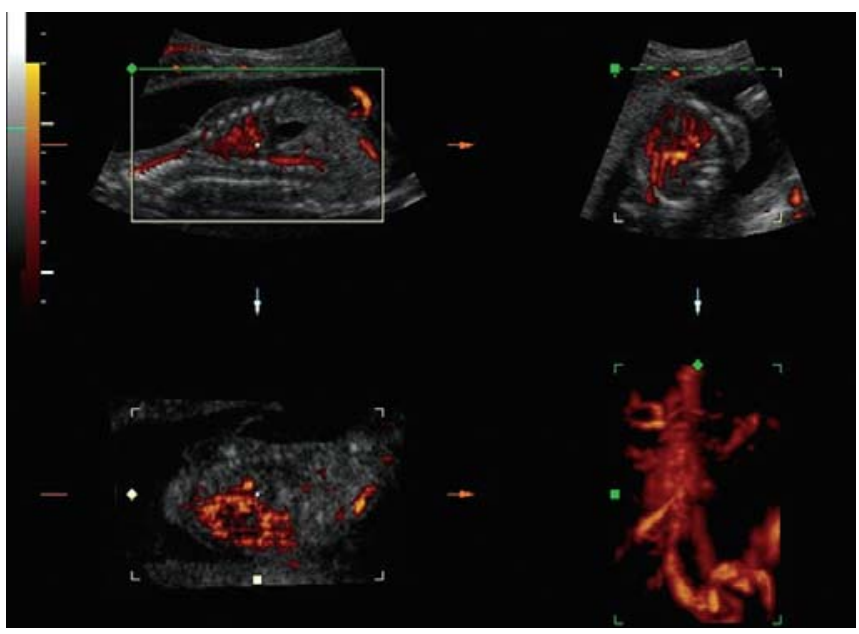

Fig. 8: Fetal circulation at 14 weeks' gestation demonstrated by power Doppler and gray-scale three-dimensional reconstruction. (From Embryology Image Library at Paul L Foster School of Medicine, Editors: Sanja Plavsic Kupesic, MD, PhD and Guillermo Azumendi, MD)

is released in response to hypoxic tissue and stimulated new capillary growth. Hyperoxic conditions decrease VEGF levels resulting in a "pruning" of newly developed vessels. Later, the vessels stabilize and are no longer dependent on VEGF for their survival. This process of vascular development and growth continues as the fetus matures. Obviously, rapid growth of the fetus also requires rapid growth of the vascular system, the development of more RBC's and the maturation and growth of the heart.

\section{PARTURITION}

The culmination of a successful pregnancy is parturition. This process is completed many thousand times a day, yet

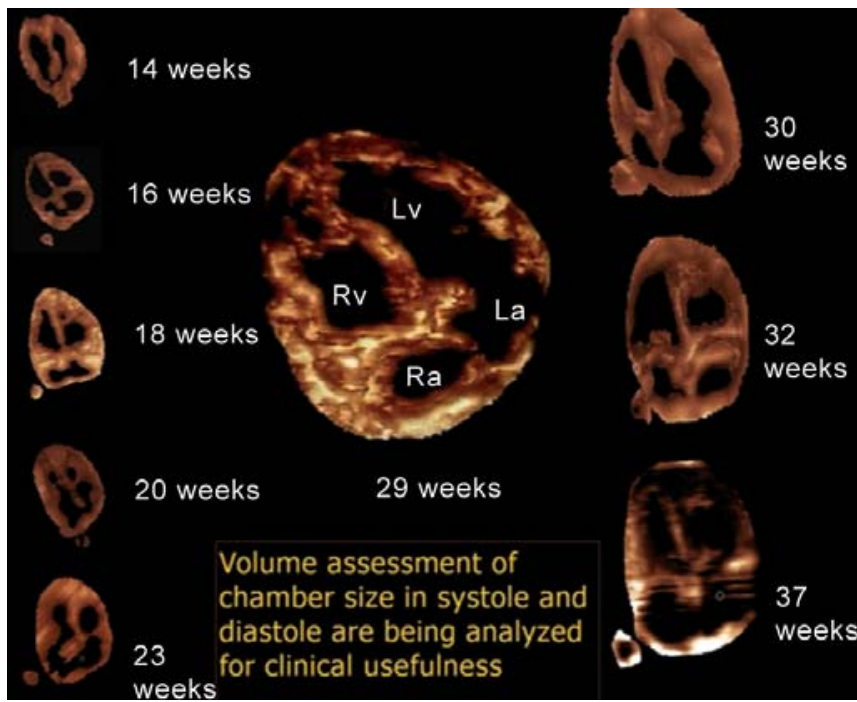

Fig. 9: Volume assessment of the fetal heart at different stages of fetal development (Courtesy of Dr Jackson DN) ${ }^{4}$

it is poorly understood. We do know that during the last weeks of the pregnancy estrogen levels rise and progesterone levels decrease. This shift in the balance of these two hormones appears to tip the scales in favor of parturition. This shift does not appear to occur rapidly, but rather develops gradually. The cause of this shift is unknown, but corticotropin releasing hormone $(\mathrm{CRH})$ is theorized to be involved. It has been suggested that this may arise from the fetus; however, maternal levels also appear to increase dramatically in the later stages of pregnancy. The rise in maternal levels of $\mathrm{CRH}$ appear to result from a decrease in corticotropin releasing hormone binding protein (CRH-BP) rather that an increase in CRP release. For unknown reasons, the CRH-BP levels decrease as $\mathrm{CRH}$ production from the placenta is increasing. This leads to a stimulation of prostaglandin production that helps to prepare the myometrium and cervix for parturition.

\section{REFERENCES}

1. Johnson LR. Essential Medical Physiology (3rd ed). Elsevier: Philadelphia 2003;47:757-75.

2. Guyton AC, Hall JE. Text book of Medical Physiology (11th ed). Elsevier: Philadelphia 2006;82:1011-41.

3. Schoenwolf GC, Bleyl SB, Brauer PR, Francis-West PH. Larsen's Human Embryology. Elsevier: Philadelphia. 2009.

4. Jackson DN, Thompson K, Slakovic M: Feasibility of Rapid Three-dimensional Imaging of the Fetal Heart. Journal of Ultrasound in Medicine March 2002;21(3). 